\title{
Editorial Introduction: Selected Papers from the 2nd Workshop on Logic, Rationality and Interaction (LORI-II)
}

\author{
Eric Pacuit
}

Received: 25 April 2012 / Accepted: 26 April 2012 / Published online: 22 May 2012

(C) The Author(s) 2012. This article is published with open access at Springerlink.com

The Second International Workshop on Logic, Rationality and Interaction (LORI-II) took place in Chongqing, China on October 8-11, 2009. ${ }^{1}$ The workshop featured a distinguished roster of invited speakers, refereed contributed papers and posters, and tutorial sessions, as well as cultural events and excursions (see http://loriweb.org/lori2009 for more information about the event). Researchers from across the world and different academic disciplines gathered in Chongqing to exchange ideas and explore new contacts between logic, mathematics, philosophy, computer science, linguistics and cognitive science. The articles in this special issue were selected from among the papers presented at the workshop [1] and the LogICCC ${ }^{2}$ meeting held on October 7th, 2009 in Chongqing. Each of these papers were extensively revised and extended after a second round of reviews. The result is a collection of papers that provides a glimpse of the many different ideas and high-quality discussions found at LORI-II.

The first paper is "Towards a Dynamic Logic of Questions" by Johan van Benthem and Ştefan Minică. This paper contributes to the growing literature on logical models of questions. Van Benthem and Minică develop a dynamic epistemic logic where questions are first-class citizens. The underlying models are based on the standard semantic interpretation of questions as partitions over a set of states. Epistemic models are extended with such a relation which

\footnotetext{
${ }^{1}$ By now there has already been a third LORI workshop. See http://golori.org for information on the LORI workshop series which takes place at various locations in China every two years.

${ }^{2} \operatorname{LogICCC}$ was a Eurocores project that funded research projects focused on logic in the Humanities, Social and Computational Sciences.
}

E. Pacuit $(\bowtie)$

Department of Philosophy, University of Maryland, College Park, MD 20742, USA

e-mail: E.J.Pacuit@uvt.nl 
is intended to represent the current "issue" to be resolved. Questions are then viewed as "triggers for explicit events of issue management". They provide a complete logic for the actions of raising, refining and resolving an issue.

Denis Bonnay and Dag Westerståhl offer a new perspective on a classic problem in the philosophy of logic in their paper "Consequence Mining: Constants versus Consequence Relations". Bolzano initiated a program of extracting a consequence relation from truth preservation patterns under replacement given a fixed set of distinguished constants [2]. Bonnay and Westerståhl propose an inverse to this construction. They start with a consequence relation and extract a set of logical constants. They find conditions that ensure there is a Galois connection between (ordered) sets of consequence relations and sets of sets of constants (ordered by inclusion).

The next paper contributes to the classic theory of belief revision initiated by Alchourrón, Gärdenfors and Makinson [3]. In "A General Family of Preferential Belief Removal Operators", Richard Booth, Thomas Meyer and Chattrakul Sombattheera study a general framework for reasoning about belief removal. The central idea is to use two relations when modeling belief change: an ordering $x<y$ which is intended to mean "world $x$ is strict more plausible than $y$ " and a second ordering $x \prec y$ which is intended to mean "the agent has an explicit reason to hold $x$ more plausible than $y$ ". They study the situation when the underlying orderings are not necessarily complete, allowing incomparibilities between worlds. The resulting class of belief removal functions is axiomatized and they show that it includes an important family of removal functions based on finite prioritized belief bases.

Robert Aumann proved a fascinating theorem in 1976 [4]: Suppose that $n$ agents share a common prior and have different private information. If there is common knowledge in the group of the posterior probabilities, then the posteriors must be equal (the agents cannot agree to disagree). Cédric Dégremont and Olivier Roy generalize this seminal result in their paper "Agreement Theorems in Dynamic-Epistemic Logic". They develop a framework for reasoning about such "agreement theorems" using epistemicplausibility models. Studying Aumann's theorem in this context raises a number of interesting technical questions (eg., common priors is not expressible in the language commonly used to reason about epistemic-plausibility models). An appropriate modal logic is defined and used to formally derive Aumann's original theorem. Their main contribution is to initiate the study of dynamic agreement results on epistemic-plausibility models, providing a new form of agreements via the operation of public announcement.

Finally, Ming Xu continues his work using the STIT-framework [5] to reason about actions in "Actions as Events". Xu starts by developing a theory of "token" actions based on his notion of an event in a branching time model. A simple theory of event composition is developed. This basic theory of events, actions and event composition is then used to define various kinds of group actions, ranging from instantaneous group actions to sequential group actions. An extended STIT-theory of agency is proposed where STIT-operators are 
combined with explicit action descriptions. Xu discusses a number of fascinating technical and conceptual issues that arise in this setting.

I would like to thank the reviewers for their hard work and the authors for their patience during such a long editorial process.

Open Access This article is distributed under the terms of the Creative Commons Attribution License which permits any use, distribution, and reproduction in any medium, provided the original author(s) and the source are credited.

\section{References}

1. He, X., Horty, J., \& Pacuit, E. (Eds.) (2009). Proceedings of the Logic, Rationality, and Interaction second international workshop, LORI 2009, Chongqing, China, 8-11 Oct 2009. Lecture notes in computer science (Vol. 5843) Springer.

2. Bolzano, B. (1837). Wissenschaftslehre. English edition: Berg, J. (1973). Theory of science (trans: Terrell, B.). Dordrecht: D. Reidel.

3. Alchourrón, C., Gärdenfors, P., \& Makinson, D. (1985). On the logic of theory change: Partial meet contraction and revision functions. Journal of Symbolic Logic, 50(2), 510-530.

4. Aumann, R. (1976). Agreeing to disagree. Annals of Statistics, 4, 1236-1239.

5. Belnap, N., Perloff, M., \& Xu, M. (2001). Facing the future: Agents and choice in our indeterminist world. Oxford: Oxford University Press. 